\title{
Artificial intelligence (AI) will enable improved diagnosis and treatment outcomes
}

\author{
Lois Holloway ${ }^{1,2,3,4} \cdot$ Eva Bezak $^{5,6} \cdot$ Clive Baldock $^{7}$
}

Accepted: 12 July 2021 / Published online: 9 August 2021

๑) Australasian College of Physical Scientists and Engineers in Medicine 2021

\section{Introduction and overview: Clive Baldock, moderator}

In recent years, artificial intelligence (AI) has increased its prevalence in society with the potential to transform many aspects of our lives. AI is considered to be not just one but a number of technologies. Based on a combination of machine learning, neural networks, deep learning and natural language processing, AI has been demonstrated to perform better than humans in certain circumstances. With the increase in size and complexity of available data, the application of $\mathrm{AI}$ and related technologies specifically to medicine and healthcare provides possibilities for contributing to significant improvements in patient outcomes with regards to diagnosis and treatments. Questions remain however as to the extent to which human beings will be replaced by AI. With its potential to be transformation in medicine and healthcare, in this topical debate Lois Holloway and Eva Bezak debate the important evolving field of AI.

Clive Baldock

cbaldock@ouw.edu.au

1 Liverpool and Macarthur Cancer Therapy Centres and Ingham Institute, Liverpool, NSW, Australia

2 South Western Sydney Clinical School, University of New South Wales, Sydney, NSW, Australia

3 Institute of Medical Physics, University of Sydney, Sydney, NSW, Australia

4 Centre for Medical Radiation Physics, University of Wollongong, Wollongong, NSW 2522, Australia

5 Cancer Research Institute and School of Health Sciences, University of South Australia, GPO BOX 2471, Adelaide, SA 5001, Australia

6 School of Physical Sciences, University of Adelaide, North Terrace, Adelaide, South Australia 5005, Australia

7 Research and Innovation Division, University of Wollongong, Wollongong, NSW 2522, Australia
Arguing for the proposition is Lois Holloway PhD. Associate Prof Lois Holloway leads the medical physics research group at the Ingham Institute and Liverpool and Macarthur Cancer Therapy centres. She is an Australasian College of Physical Scientists and Engineers in Medicine (ACPSEM) certified radiation oncology medical physicist with a strong interest in modelling to improve outcomes for cancer patients. Her current focus includes leading the Australian computer assisted theragnostics (AusCAT) distributed data network which links datasets across radiation oncology centres and the Australian MRI-linac program.

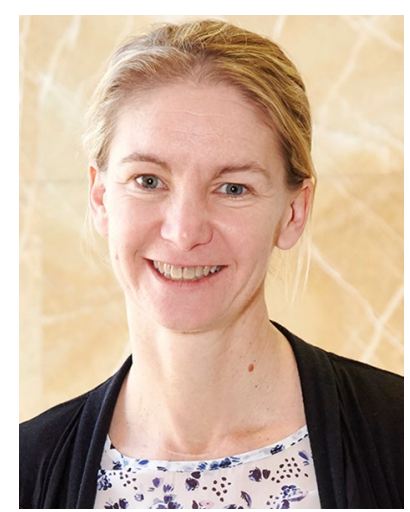

Lois Holloway

Arguing against the proposition is Eva Bezak PhD. Professor Bezak is an internationally recognised expert in the field of radiation therapy, radiation biology and radiobiological modelling, e.g. for proton-therapy of pediatric brain tumours, development of novel radiopharmaceuticals for targeted-alpha-therapy for pancreatic cancer. She has held numerous leadership positions, including Chief Medical Physicist, Royal Adelaide Hospital (2007); Professor in Medical Radiation, University of South Australia (UniSA) (2015), Director; Translational Cancer Research Centre, Cancer Research Institute, UniSA (2018), Professorial Lead, UniSA (2020), Vice President Asia-Oceania Federation of 
Organizations for Medical Physics (AFOMP), Secretary General International Organization for Medical Physics (IOMP), and others. She has published 3 books, over 160 papers and supervised over 35 Higher Degree by Research (HDR) students.

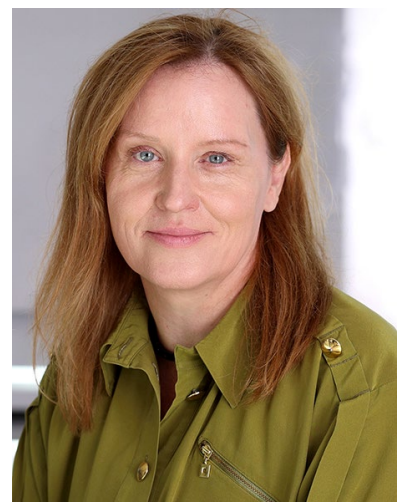

Eva Bezak

\section{For the proposition: Lois Holloway}

\section{Opening statement}

$\mathrm{AI}$ is the ability of a computer to perform tasks commonly associated with intelligent beings and frequently includes the ability to reason and learn from past experience [1]. The question of this topical debate is can AI enable improved diagnosis and treatment outcomes. My immediate, non-validated, response is 'yes!' and with so many of us in academia, clinics and business focusing resources to develop, validate and implement AI [2], it would seem so does our community. However, let's take a step back and look at the evidence.

Let's start with the low hanging fruit; the challenges of workflow, safety and limited resources. In any clinic, there is a desire to be able to do more for our patients, through improved efficiencies, accuracy, precision and safety. AI enables improved outcomes in two ways; (i) Where there are high resources, boundaries can be pushed to improve outcomes; e.g. automated tracking with MRIlinac guidance in radiotherapy and (ii) Where there are low resources, AI systems allow workflow improvements; e.g. automated segmentation [3] and safer treatments [4].

Secondly, let's consider the ability to detect factors not obvious to humans, either due to sheer volume of data or the nature of the factors or patterns. There is an abundance of evidence of well validated $\mathrm{AI}$ algorithms to detect these factors and importantly, now growing evidence that these algorithms have impact in the clinic; e.g. improved reading and diagnosis in mammography with AI support [5], dose adaptions for inflammatory bowel disease [6] and even predicting two year survival for lung cancer [7].

Thirdly, and as importantly, AI enables AI thus providing us with access to the mountains and mountains of electronic data that we are accruing each and every day in our clinics. In a manual fashion it is close to impossible to extract and review all of this data in a timely fashion, let alone determining and validating patterns within this data. AI provides us with the infrastructure and framework to enable access to this wide and varied data [8] such that we can learn patterns in rare diseases and learn rare insights from more common diseases. In this instance the infrastructure provided by AI, enables AI and thus further improvements in diagnosis and treatment outcomes.

The ability to reason and learn from past experience, as well as a core component of AI, are the key goals of our professions. It is this ability to reason and learn from past experience that has enabled our professions to have so much impact on diagnosis and treatment outcomes to date. AI tools enable us to fulfil the core goals of our professions by providing efficiency and effectiveness gains as demonstrated with evidence in this short commentary. Thus, just as our professions have enabled improved diagnosis and treatment outcomes, so too will the addition of (greater use of) AI in our practices.

\section{Against the proposition: Eva Bezak}

\section{Opening statement}

A reliable AI tool in medicine must be broadly trained (however, the literature on AI tool training shows data sets of 10-30 participants or participants of 1 ethnicity only). There is evidence that, e.g. AI algorithms for detection of benign and malignant moles were more accurate in fair-skinned people but underperformed in diagnosing lesions in skins of colour, due to training on datasets containing predominantly fair-skinned patients [9]. According to Adamson [9] and Crawford [10], "blind spots in ML reflect the worst societal biases". Hence, establishment of national and international registries to ensure data collection across genetically and racially diverse populations is a must. However, this can take a long time due to local policies, ethics regulations, bureaucracy, etc., compounded by the fact that most AI algorithms need access to massive datasets to develop robust algorithms with a sufficiently low generalisability error. However, it is still unclear as to what the optimal size of training sets needs to be.

Data Privacy and security: patient awareness that their information may (even anonymously) be used in the design, 
training or testing of $\mathrm{AI}$ is often not considered in their consent. Interestingly, 55\% of consumers in a recent survey do not trust tech companies to keep digital health information secure [11]. As medical data sets for use in AI applications are created through implementation of electronic record systems (clinical notes, pathology and imaging, PACS), in addition to patient privacy issues, this also raises cyber-security issues [12].

To share sensible information, standardized data needs to be collected, requiring consensus nationally and internationally. Unfortunately, it is not completely clear what knowledge/data should be recorded and used. Additionally, the volume and variability of data mean it is hard to translate all this data into knowledge guiding clinical decisions [13]. For example, radiology reports are often unstructured and the extracted information has a relatively low quality. A study has found that $2-20 \%$ of radiology reports contain demonstrable errors [14].

Do we have sufficient storage for all this data that is bound to increase greatly? Global data generation in 2010 was 2 Zeta-Bytes (ZB) and was expected to increase to over $50 \mathrm{ZB}$ by 2020 and $175 \mathrm{ZB}$ by 2025 [15]. We will definitely need quantum computing - but we are not there as yet.

From radiology, radiation oncology point of view, where AI heavily utilizes image data sets, there is an issue of image variability between imaging equipment from different manufacturers, different generations of equipment or different imaging protocols, raising concerns about reliability of AI systems. Lastly, there is uncertainty and lack of clear guidelines on AI clinical implementation, validation, and quality assurance.

The 'big data' demands of the systems result in complex ethical considerations [16]. Regulatory bodies worldwide must develop suitable control measures and guidelines. A hotly debated ethical question is the responsibility in case of misdiagnosis done by an AI system. The question is whether AI should be used as a system on its own or should it be a tool used by physicians, where responsibility lies with the physicians themselves [16].

In summary, "many stars will have to align" before AI will be able to reliably and ethically improve patient diagnosis and treatment outcomes.

\section{For the proposition: Lois Hollowy}

\section{Rebuttal statement}

So will 'many stars' really 'have to align before AI will be able to reliably and ethically improve patient diagnosis and treatment outcomes'? There certainly are many challenges to developing and implementing $\mathrm{AI}$, as presented in the opposing opening statement. However, does this negate the fact that AI will enable improved diagnosis and treatment outcomes or as I've previously suggested that it has enabled this already [3-7]?

Cyber security is not an issue for AI alone, we are all working to ensure effective and safe computing functionality. AI has the potential to provide solutions to this issue, ensuring greater cyber security for both AI development and our clinical and other systems [17].

Data storage and ethics considerations must be carefully considered, but these challenges have resulted in some very innovative solutions to date [18] and although international standardisation will not come quickly, project based solutions have demonstrated feasibility. It should also be noted that the majority of international ethics guidelines consider quality improvement activities on retrospective health data within ethical constraints of the data collected. In fact, it could be considered unethical (lacking merit, integrity, justice, benefice, respect; the values and principles of national statements on human research ethics) to not use this data for improvement in diagnostic and treatment outcomes.

Standardised data collection would be ideal for AI algorithms. However, there is data to suggest that a broad range of data may be more valuable than standardisation. AI can also be used to account for the lack of standardisation, adapting imaging data to overcome these challenges and ensuring applicability of developed AI tools to a broad range of centres and patients $[19,20]$.

Finally, it's hard to argue against the fact that AI will improve diagnosis and treatment outcomes when, as demonstrated in my opening statement, in many instances this is already the case [3-7]. As a community we need to continue to work together to safely and effectively ensure that we take full advantage of improvements in diagnosis and treatment outcomes using AI.

\section{Against the proposition: Eva Bezak}

\section{Rebuttal statement}

While the premise and the promise of AI sound appealing, exciting, offering potential solutions from retail, through traffic management to medicine, it is still a long way before we can conclude that AI, in the field of healthcare, will improve diagnosis and treatment outcomes. I observe with concern that the speed of discovery, application development and implementation are unprecedented and timelines for research translation are shortened exponentially, potentially increasing risks of incidents or failures. And it is this speed that may also be the AI's biggest enemy, since entire systems of current clinical practice, management and governance must be transformed to ensure safe implementation of AI into healthcare [21]. 
The author of the affirmative statement has made herself some conditional statements in regard to AI, e.g.: A) well-validated data-it is not clear how large and diverse the datasets should be and how they should be Qa-ed and validated; B) sheer volume of data (going back to standardization, storage, interpretation, data quality); C) the challenges of workflow, safety and limited resources-this works both ways and evidence shows that there are workforce requirements to collect and input large amount of information; or safe implementation of AI.

Finally, there are a number questions that we need to address in our professions, including [21, 22]:

- How do we implement big data and artificial intelligence into clinics?

- How are our professional roles changing?

- How do we need to adjust our training and/or ongoing professional development?

- How can engineers, physicists and IT specialists work together with clinicians and healthcare providers to ensure safe adoption and implementation of these new technologies?

I have to conclude again, we need to tread carefully, to ensure that AI and all associated processes are developed and implemented safely and for benefits of our patients.

\section{References}

1. Copeland BJ (2020) Artifical Intelligence. Encyclopedia Britannica. https://www.britannica.com/technology/artificial-intelligen ce. Accessed 3 Jan 2021

2. He J, Baxter SL, Xu J et al (2019) The practical implementation of artificial intelligence technologies in medicine. Nat Med 25(1):30-36

3. Zabel WJ, Conway JL, Gladwish A et al (2021) Clinical evaluation of deep learning and atlas based auto-contouring of bladder and rectum for prostate radiotherapy. Pract Radiat Oncol 11:e80-e89

4. Choudhury A, Asan O (2021) Role of Artificial Intelligence in Patient Safety Outcomes: Systematic Literature Review. JMIR Med Inform 8(7):e18599

5. Rodríguez-Ruiz A, Krupinski E, Mordang J et al (2019) Detection of breast cancer with mammography: effect of an artificial intelligence support system. Radiology 290(2):305-314

6. Strik AS, Löwenberg M, Mould DR et al (2021) Efficacy of dashboard driven dosing of infliximab in inflammatory bowel disease patients; a randomized controlled trial. Scand J Gastroenterol 56(2):145-154

7. Oberije C, Nalbantov G, Dekker A et al (2014) A prospective study comparing the predictions of doctors versus models for treatment outcome of lung cancer patients: a step toward individualized care and shared decision making. Radiother Oncol 112(1):37-43

8. Deist TM, Dankers FJWM, Ojha P et al (2020) Distributed learning on $20000+$ lung cancer patients - the personal health train. Radiother Oncol 144:189-200

9. Adamson AS, Smith A (2018) machine learning and health care disparities in dermatology. JAMA Dermatol 154(11):1247-1248

10. Crawford K (2016) Artificial Intelligence's White Guy Problem. New York Times. https://www.nytimes.com/2016/06/26/opinion/ sunday/artificial-intelligences-white-guy-problem.html. Accessed 3 Jan 2021

11. Accenture (2020) Re-examining the Accenture 2020 Digital Health Consumer Survey. How can leaders make recent digital health gains last? US Findings. https://www.accenture.com/_ acnmedia/PDF-130/Accenture-2020-Digital-Health-ConsumerSurvey-US.pdf\#zoom=40. Accessed 3 Jan 2021

12. Finlayson SG, Bowers JD, Ito J (2019) Adversarial attacks on medical machine learning. Science 363(6433):1287-1289

13. Marcu LG, Boyd C, Bezak E (2019) Current issues regarding artificial intelligence in cancer and health care. Implications for medical physicists and biomedical engineers. Health Technol 9(4):375-381

14. Brady A, Laoide RO, McCarthy P et al (2012) Discrepancy and error in radiology: concepts, causes and consequences. Ulster Med J 81(1):3-9

15. Reinsel D, Gantz J, Rydning J (2018) Data Age 2025: The digitization of the world: From edge to core. https://resources.mored irect.com/white-papers/idc-report-the-digitization-of-the-worldfrom-edge-to-core. Accessed 1 Apr 2021

16. Paulson SS, Scruth E (2017) Legal and ethical concerns of big data: predictive analytics. Clin Nurse Spec 31(5):237-239

17. Zeadally S, Adi E, Baig Z et al (2020) Harnessing artificial intelligence capabilities to improve cybersecurity. IEEE Access 8:23817-23837

18. Jochems A, Deist TM, van Soest J et al (2016) Distributed learning: developing a predictive model based on data from multiple hospitals without data leaving the hospital - A real life proof of concept. Radiother Oncol 121(3):459-467

19. Traverso A, van Soest J, Wee L et al (2018) The radiation oncology ontology (ROO): publishing linked data in radiation oncology using semantic web and ontology techniques. Med Phys 45(10):e854-e862

20. Zhovannik I, Bussink J, Traverso A et al (2019) Learning from scanners: bias reduction and feature correction in radiomics. Clin Trans Radiat Oncol 19:33-38

21. Cath C, Wachter S, Mittelstadt B (2018) Artificial intelligence and the "good society": the US, EU, and UK approach. Sci Eng Ethics 24(2):505-528

22. Kortesniemi M, Tsapaki V, Trianni A (2018) The European federation of organisations for medical physics (EFOMP) white paper: big data and deep learning in medical imaging and in relation to medical physics profession. Physica Med 56:90-93

Publisher's Note Springer Nature remains neutral with regard to jurisdictional claims in published maps and institutional affiliations. 